\title{
Dynamic Behavior and Stability Analysis of Nonlinear Modes in the Fourth Order Generalized Ginzburg-Landau Model with Near PT-symmetric Potentials
}

\author{
Jiarui Zhang \\ China Agricultural University \\ Jiaqi Zhang \\ China Agricultural University College of Engineering \\ Zhaolin Zheng \\ China Agricultural University \\ Da Lin \\ China Agricultural University \\ Yujia Shen ( $\nabla$ yjshen2018@cau.edu.cn ) \\ China Agricultural University
}

\section{Research Article}

Keywords: generalized Ginzburg-Landau equation, near PT -symmetric potential, stable nonlinear mode, soliton and peakon solutions

Posted Date: January 25th, 2022

DOI: https://doi.org/10.21203/rs.3.rs-1205046/v1

License: (c) (i) This work is licensed under a Creative Commons Attribution 4.0 International License.

Read Full License 


\title{
Dynamic behavior and stability analysis of nonlinear modes in the fourth order generalized Ginzburg-Landau model with near $\mathcal{P} \mathcal{T}$-symmetric potentials
}

\author{
Jia-Rui Zhang · Jia-Qi Zhang • Zhao-Lin Zheng • \\ Da Lin • Yu-Jia Shen
}

Received: date / Accepted: date

\begin{abstract}
We investigate the fourth order generalized Ginzburg-Landau equation (GGLE) which can be widely used in hydrodynamics and nonlinear optics. There are three major ingredients. Firstly, bilinear form of the fourth order GGLE is obtained by means of Hirota method. Then we get the analytical soliton solution with different dynamic behaviors in two cases, corresponding to the constant and variable coefficients respectively. Secondly, some numerical simulations with near $\mathcal{P} \mathcal{T}$-symmetric potentials are carried out. When the potential is closer to conventional $\mathcal{P} \mathcal{T}$ symmetric potential, the nonlinear modes tend to unstable. But by increasing the value of imaginary part of potentials, It can be found that the amplitude of nonlinear mode is periodically oscillating. Thirdly, we consider the excitations of nonlinear modes and get some stable cases that have not been acquired in second part.
\end{abstract}

Keywords generalized Ginzburg-Landau equation · near $\mathcal{P} \mathcal{T}$-symmetric potential · stable nonlinear mode $\cdot$ soliton and peakon solutions

Mathematics Subject Classification (2000) $37 \mathrm{~K} 40 \cdot 35 \mathrm{C} 08$

\section{Introduction}

With the development of symbolic computation and soliton theory, the nonlinear evolution equations have become more interesting in hydrodynamics and nonlinear optics [1-4], in which the complex Ginzburg-Landau models have riveted the attention of researchers recently. Moreover, the conventional Ginzburg-Landau equation (GLE) [5-7]

$$
i u_{t}+p u_{x x}+q|u|^{2} u=i \gamma u
$$

is one of the universal nonlinear wave models. It has been widely used in superfluidity, plasmas, Bose-Einstein condensation, liquid crystals, strings in the field theory, quantum field theory and etc. [8-10]. As dissipative extensions of the conservative nonlinear Schrödinger equation, various

J.-R. Zhang · Z.-L. Zheng · D. Lin · Y.-J. Shen*

College of Science, China Agricultural University, Beijing 100083, China

E-mail: yjshen2018@cau.edu.cn

J.-Q. Zhang

College of Engineering, China Agricultural University, Beijing 100083, China 
types of soliton solutions of GLE are analyzed in detail, including multi-peak solitons [11], exploding solitons [12], two-dimensional vortical solitons [13], lattice solitons [14] and peakons. Although the higher order GLEs have been investigated, nonlinear modes of them have rarely been analyzed so far. Therefore, the main purpose of this paper is to study the generalization of GLE with fourth order nonlinear dispersion by means of analytical and numerical methods.

We will investigate the fourth order generalized GLE:

$$
\begin{aligned}
& i u_{t}+\alpha(x) u_{x x}+\beta(x, t)|u|^{2} u+\gamma(x, t) u+\sigma(t) u_{x x x x}+\zeta(t) u_{x x}|u|^{2} \\
& +\xi(t) u|u|_{x x}^{2}+\rho(t) u|u|^{4}+\eta(t) u_{x}=0,
\end{aligned}
$$

with $\alpha=\alpha_{1}+i \alpha_{2}, \beta=\beta_{1}+i \beta_{2}, \gamma=V+i W, \sigma=\sigma_{1}+i \sigma_{2}, \zeta=\zeta_{1}+i \zeta_{2}, \xi=\xi_{1}+i \xi_{2}, \rho=\rho_{1}+i \rho_{2}$, $\eta=i \eta_{1}$, where $V, \alpha_{i}(i=1,2)$ are real functions of $x ; \eta_{1}, \sigma_{i}, \zeta_{i}, \xi_{i}, \rho_{i}(i=1,2)$ are real functions of $t ; W, \beta_{i}(i=1,2)$ are real functions of $x$ and $t$. To indicate the the linear gain-loss coefficient, we introduce the complex potential $V+i W[8]$.

Eq. (2) can be applied to describing propagation of light in an active dispersive medium [15]. In this case, $u$ is the the complex envelope of the electric field, $x$ denotes the propagation distance, and $t$ is the retarded time. $\alpha_{1}$ is the the group velocity dispersion coefficient, and $\alpha_{2}$ describes the spectral filtering or linear parabolic gain [12]. $\beta_{1}$ is Kerr-nonlinearity coefficient and $\beta_{2}$ accounts for the nonlinear gain-loss processes [8]. $\rho_{1}$ is the parameter of the quintic nonlinearity and represents a higher order correction to the nonlinear amplification/absorption, and $\rho_{2}$ characterizes the saturation of the nonlinear gain and it is a possible higher order correction term to the intensity-dependent refractive index [15]. $\sigma$ represents the effect due to discreteness and higher order magnetic interactions [16]. $V$ is related to the refractive index waveguide, and $W$ characterizes the amplification (gain) or absorption (loss) of light beam in the optical material $[8,17]$.

Put forward by Bender and his coworker in 1998 [18-20], $\mathcal{P} \mathcal{T}$ behaviors have attracted considerable attention in optical experiments [21-29]. In recent years, there has been tremendous interest in investigating one- and multi-dimensional solitons and their stability in all stripes of optical potentials $[3,30,23,25]$. In addition, stable solitons in the nonlinear Schrödinger equation with some non- $\mathcal{P} \mathcal{T}$-symmetric potentials have obtained extensive research $[31-37,4]$. The non- $\mathcal{P} \mathcal{T}$-symmetric potentials can be bifurcated out from the $\mathcal{P} \mathcal{T}$-symmetric potential by regulating the related potential parameters, which are called the near $\mathcal{P} \mathcal{T}$-symmetric potentials [8]. This paper is to investigate Eq. (2) with the near $\mathcal{P} \mathcal{T}$-symmetric potentials to obtain the stationary nonlinear modes.

There are three special cases of Eq. (2) that should be mentioned:

(1)When $\alpha, \beta, \gamma$ are constants and the other coefficients are zero, Eq. (2) is degenerated to Eq. (1). The stable optical soliton can be gotten by regulating the group velocity dispersion and nonlinear gain-loss coefficient [38]. Moreover, many kinds of analytical coherent structure solutions of this equation have been studied [39].

(2) When $\alpha=\beta=1, \gamma(x, t)=V(x)+i W(x)$ and the other coefficients are zero, Eq. (2) can be reduced to

$$
i u_{t}+u_{x x}+[V(x)+i W(x)] u+|u|^{2} u=0,
$$

in which the beam evolution is governed by the normalized nonlinear Schrödinger-like equation. It can be used to describe the propagation of the optical soliton in a self-focusing Kerr nonlinear $\mathcal{P} \mathcal{T}$-symmetric potential $[40]$.

(3)When $\alpha, \beta$ are complex constants, $\gamma(x, t)=V(x)+i W(x)$ and the other coefficients are zero, Eq. (2) can be reduced to

$$
i u_{t}+\left(\alpha_{1}+i \alpha_{2}\right) u_{x x}+[V(x)+i W(x)] u+\left(\beta_{1}+i \beta_{2}\right)|u|^{2} u=0,
$$

which can describe the spatial beam transmission in a cubic-nonlinear optical medium described by the complex Ginzburg-Landau equation with complex potentials. Moreover, the stability of soliton has been analyzed via numerical simulation [8]. 
In Sect. 2, under some constraints of the constant and variable coefficients that will be derived, Eq. (2) will be bilinearized. Based on the bilinear forms, one-soliton solutions will be derived. In Sect. 3, we will perform numerical simulation of Eq. (2) with near $\mathcal{P} \mathcal{T}$-symmetric potentials in two cases, corresponding to the near $\mathcal{P} \mathcal{T}$-symmetric Scarf-II and $\delta$-signum potentials, respectively. Then we consider excitations of solitons via making the parameters rely on the propagation distance $t$. In, Sect. 4, results will be summarized.

\section{ANALYTICAL SOLITON SOLUTIONS OF EQ. (2)}

To get the bilinear forms of Eq. (2), we will present the following constraints on the variable coefficients:

$$
\alpha(x) \beta(x, t) \zeta(t)=6 \beta^{2}(x, t) \sigma(t)=6 \alpha(x) \beta(x, t) \xi(t)=2 \alpha^{2}(x) \rho(t) .
$$

Via the dependent-variable transformation

$$
u(x, t)=\frac{g(x, t)}{f(x, t)},
$$

with the real $f$ and complex $g$, we can derive the bilinear forms of Eq. (2):

$$
\begin{aligned}
& i D_{t} g \cdot f+\alpha(x) D_{x}^{2} g \cdot f+\gamma(x, t) g \cdot f+\sigma(t) D_{x}^{4} g \cdot f+\eta(t) D_{x} g \cdot f=0, \\
& \alpha(x) D_{x}^{2} f \cdot f-\beta(x, t)|g|^{2}=0 .
\end{aligned}
$$

Through Hirota bilinear method, we introduce the bilinear operator $D$ defined as [41]

$$
D_{x}^{m} D_{t}^{n} a(x, t) \cdot b(x, t)=\left.\frac{\partial^{m}}{\partial y^{m}} \frac{\partial^{n}}{\partial s^{n}} a(x+y, t+s) b(x-y, t-s)\right|_{y=0, s=0},
$$

where $m, n$ are positive integers, $a, b$ are functions of $x$ and $t$, and $y$ stands for the small increment.

Then we expand $g$ and $f$ in power series of a small parameter $\epsilon$ as

$$
\begin{aligned}
& g=\epsilon g_{1}+\epsilon^{3} g_{3}+\epsilon^{5} g_{5}+\cdots, \\
& f=1+\epsilon^{2} f_{2}+\epsilon^{4} f_{4}+\epsilon^{6} f_{6}+\cdots,
\end{aligned}
$$

where $g_{m}(m=1,3,5, \cdots), f_{n}(n=2,4,6, \cdots)$ are functions of $x$ and $t$ to be determined.

2.1 The case of constant coefficients

In this section, we assume the coefficients of Eq. (2) are constant and set

$$
\begin{aligned}
& g_{1}=e^{k_{1} x+\left(\omega_{1}+i \omega_{2}\right) t+\theta_{1}}, \\
& f_{2}=A_{1} e^{2 k_{1} x+2 \omega_{1} t+2 \theta_{1}},
\end{aligned}
$$

where $k_{1}, A_{1}, \omega_{i}(i=1,2)$ are non-zero constants. Let $\beta_{1}=c_{0} \alpha_{1}, \beta_{2}=c_{0} \alpha_{2}, c_{0}=8 A_{1} k_{1}^{2}$, and we can derive the constraint relation of other parameters:

$$
\begin{aligned}
& \omega_{1}=-k_{1}^{2} \alpha_{2}-W-k_{1} \eta_{1}-k_{1}^{4} \sigma_{2}, \\
& \omega_{2}=k_{1}^{2} \alpha_{1}+V+k_{1}^{4} \sigma_{1}, \\
& W=-k_{1}^{4} \sigma_{2}-k_{1}^{2} \alpha_{2} .
\end{aligned}
$$


(a)

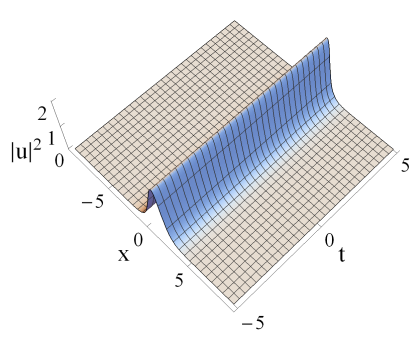

(b)

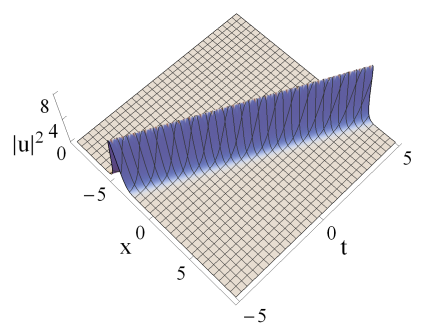

(c)

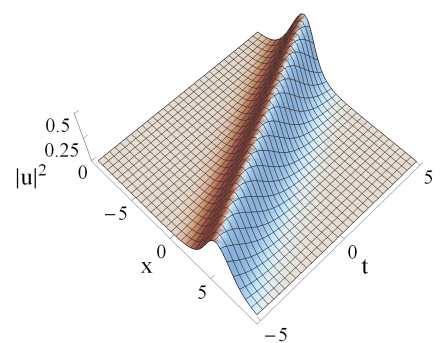

Figs. 1 Parameters are chosen as: (a) $k_{1}=1, \alpha_{2}=1, c_{0}=1, \eta_{1}=0, \theta_{1}=0$. (b) and (c) have two different parameters from (a), with $k_{1}=2, \eta_{1}=1$ in (b) and $k_{1}=0.5, \eta_{1}=-1$ in (c).

Without loss of generality, we set $\epsilon=1$. Thus, the expression of single soliton solution can be written as

$$
u=\frac{g_{1}}{1+f_{2}} .
$$

In Figs. 1, the propagation of the soliton along the distance $x$ is illustrated. The velocity and direction of solitonic propagation phase will be changed if we choose different values of $\eta_{1}$. Moreover, The amplitude of the soliton is determined by $k_{1}$.

2.2 The case of various coefficients

Next, we will consider the situation that the coefficients of Eq. (2) are functions of $x, t$ and assume

$$
\begin{aligned}
& g_{1}=A_{1}(t) \mathrm{e}^{k_{1} x+\omega_{1}(t)+i \omega_{2}(t)}, \\
& f_{2}=A_{2}(t) \mathrm{e}^{2 k_{1} x+2 \omega_{1}(t)} .
\end{aligned}
$$

Then we separate $W(x, t)$ into two parts by $W(x, t)=W_{1}(x)+W_{2}(t)$, and take $\beta_{1}(x, t)=$ $2 c_{0}(t) \alpha_{1}(x), \beta_{2}(x, t)=2 c_{0}(t) \alpha_{2}(x)$.

The expressions of variable coefficients can be obtained

$$
\begin{aligned}
& A_{1}(t)=\mathrm{e}^{\int-W_{2}(t) d t}, \\
& A_{2}(t)=\frac{A_{1}^{2}(t) c_{0}(t)}{4 k_{1}^{2}}, \\
& \omega_{1}(t)=\int-k_{1}\left(\eta_{1}(t)+k_{1}^{3} \sigma_{2}(t)\right) d t, \\
& \omega_{2}(t)=\int k_{1}^{4} \sigma_{1}(t) d t, \\
& c_{0}(t)=c_{1} \mathrm{e}^{\int 2\left(W_{2}(t)+k_{1}^{4} \sigma_{2}(t)\right) d t},
\end{aligned}
$$

where $c_{1}, k_{1}$ are constants, $V(x)=-k_{1}^{2} \alpha_{1}(x)$ and $W_{1}(x)=-k_{1}^{2} \alpha_{2}(x)$.

The analytical solution of Eq. (2) with variable coefficients can be likewise expressed as Eq. (12). In Figs. 2, we take the variable coefficient $\eta_{1}(t)$ as sine function. It is obvious that the soliton solution is periodic and $\sigma_{2}(t)$ is related to the amplitude.

When $W_{2}(t)=a t \mathrm{e}^{-t^{2}}, \eta_{1}(t)=b \mathrm{e}^{-t^{2}}, \sigma_{2}(t)=c \mathrm{e}^{-t^{2}}$, it can be seen that $a$ and $b$ have effects on the amplitude and phase shift of soliton near $t=0$ respectively. The amplitude of soliton in $t \geq 0$ is directly proportional to $c$ and the other part is inversely [see Figs. 3]. 
(a)

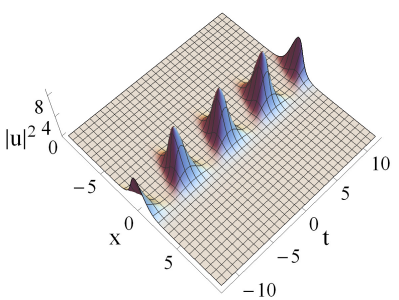

(b)

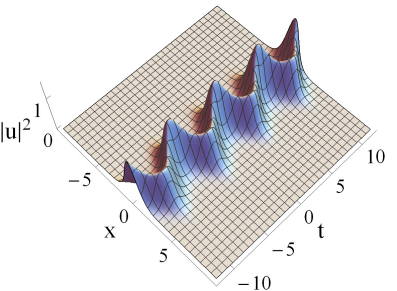

(c)

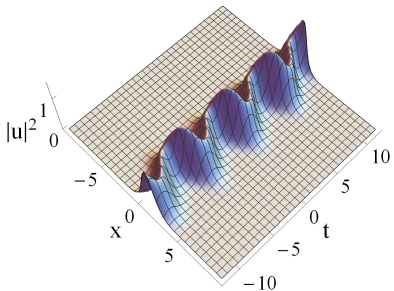

Figs. 2 Parameters are chosen as: $k_{1}=1, c_{1}=1$, with (a) $W_{2}(t)=0.1 \sin (2 t), \eta_{1}(t)=\sin (t), \sigma_{2}(t)=\sin (t)$, with (b) $W_{2}(t)=0.1 \sin (2 t), \eta_{1}(t)=\sin (t)$, with $\sigma_{2}(t)=0.1 \sin (t)$, with (c) $W_{2}(t)=0.1 \sin (2 t), \eta_{1}(t)=\cos (t)$, $\sigma_{2}(t)=0.1 \sin (t)$.

(a)

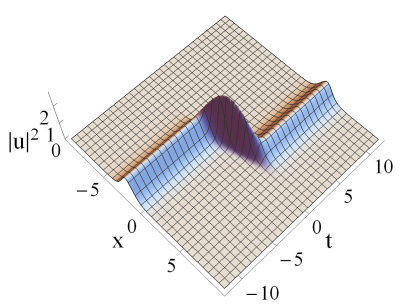

(b)

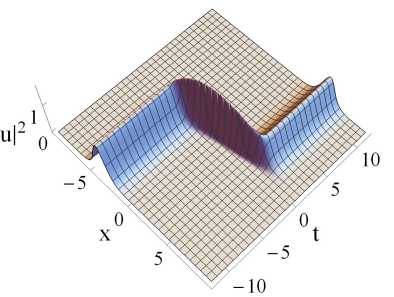

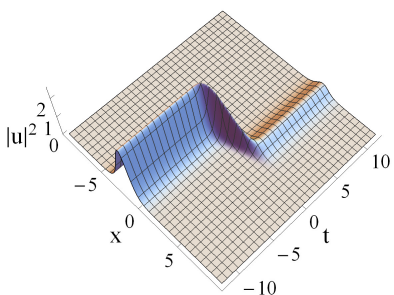

Figs. 3 Parameters are chosen as: $k_{1}=1, c_{1}=1$, with (a) $a=1, b=3, c=0.1$, with (b) $a=0.5, b=5, c=0.1$, with (c) $a=0.5, b=3, c=0.5$.

\section{ANALYSIS OF NUMERICAL SOLUTIONS}

To study the effect of fourth order nonlinear dispersion numerically, we consider the generalized case with constant coefficients and complex potentials

$$
i u_{t}+\left(\alpha_{1}+i \alpha_{2}\right) u_{x x}+\left(\beta_{1}+i \beta_{2}\right)|u|^{2} u+[V(x)+i W(x)] u+\left(\sigma_{1}+i \sigma_{2}\right) u_{x x x x}+\left(\rho_{1}+i \rho_{2}\right) u|u|^{4}=0,
$$

where $\alpha_{i}, \beta_{i}, \sigma_{i}$ and $\rho_{i}(i=1,2)$ are all real parameters. We focus on the stationary solutions of Eq. (15) in the form:

$$
u(x, t)=\phi(x) e^{-i \mu t}
$$

where $\mu$ is a real propagation constant.

Substituting it into Eq. (15), we can obtain the complex localized field-amplitude function $\phi(x)$ that satisfies the ordinary differential equation:

$$
\left[\left(\alpha_{1}+i \alpha_{2}\right) \frac{d^{2}}{d x^{2}}+\left(\beta_{1}+i \beta_{2}\right)|\phi|^{2}+V(x)+i W(x)+\left(\sigma_{1}+i \sigma_{2}\right) \frac{d^{4}}{d x^{4}}+\left(\rho_{1}+i \rho_{2}\right)|\phi|^{4}\right] \phi=\mu \phi .
$$

In our numerical simulations, spatial differential and the integration in time are carried out by modified squared-operator method and the pseudospectral method, respectively [42]. In the following, we study the Eq. (15) under the role of the near $\mathcal{P} \mathcal{T}$-symmetric Scarf-II and $\delta$-signum potentials, and find the stationary nonlinear modes of Eq. (15). 
3.1 Nonlinear modes with the near $\mathcal{P} \mathcal{T}$-symmetric Scarf-II potential

The $\mathcal{P} \mathcal{T}$-symmetric complex potential $V(x)+i W(x)$ has the features that $V(x)=V(-x)$ and $W(-x)=-W(x)$ [3]. Because of the appearance of complex coefficients, Eq. (2) is not $\mathcal{P} \mathcal{T}-$ symmetric. The near $\mathcal{P} \mathcal{T}$-symmetric potentials are considered [8]. We initiate our analysis by introducing the following near $\mathcal{P} \mathcal{T}$-symmetric Scarf-II potential

$$
\begin{aligned}
& V(x)=V_{0} \operatorname{sech}^{2}(x), \\
& W(x)=W_{0} \operatorname{sech}(x) \tanh (x)-W_{1} \operatorname{sech}^{2}(x) .
\end{aligned}
$$

After setting some coefficients, we study the effects of other variables on the iterative image. The power of nonlinear mode is defined as $P=\int_{-\infty}^{+\infty}|\phi(x, t)|^{2} d x$. The relationship between $\rho_{1}$, $\rho_{2}, \alpha_{2}, V_{0}, W_{1}$ and $P$ is shown in the Figs. $4(\mathrm{a})$ and $4(\mathrm{~b})$, in which the horizontal coordinate is $W_{1} \in(0,6)$. When other parameters are specified, $W_{1}$ and $P$ are positively correlated while $\rho_{1}, \rho_{2}$ and $P$ are negatively correlated [see Fig. 4(a)]. What's more, when $\alpha_{2}$ is different in two cases, we can make them have same power by adjusting $W_{1}$ [see Fig. $4(\mathrm{~b})$ ].

In addition, when $W_{1}=0$, the near $\mathcal{P} \mathcal{T}$-symmetric potential (18) is just the conventional $\mathcal{P} \mathcal{T}$ symmetric Scarf-II potential. With the value of $W_{1}$ decreasing, the solution will be unstable and become attenuation by the propagation of soliton. Now we consider the evolution of soliton solutions via Eq. (16). In our numerical simulations, the $5 \%$ initial random noise is added to simulate the wave transmission. With the given parameters, Fig. 4(c) displays a stable nonlinear mode. If the value of $W_{1}$ is decreased, the mode will become unstable. This is to say a little change of the gain-loss distributions can make the nonlinear mode unstable when $W_{1}$ is sufficiently small [see Fig. 4(f)].

Furthermore, It will get close to $\mathcal{P} \mathcal{T}$-symmetric Scarf-II potential by increasing the value of $W_{0}$. In this case, the relationship between $P$ and $W_{0}$ is illustrated in Fig. 5(a). If the initial value of $W_{0}=20 W_{1}$, then the amplitude of nonlinear mode is periodically oscillating and it experiences more than 2 periods within $1450 \leq t \leq 1500$ [see Fig. 5(b)]. This means the growth of $W_{0}$ can also change the stability of soliton with near $\mathcal{P} \mathcal{T}$-symmetric Scarf-II potential.

When other coefficients are fixed, we change $W_{0}$ to get different potentials. Let $W_{0}=0.1$, and $W$ approximates to an even function of $x$. Moreover, the soliton is stable and symmetric approximately [see Figs. 5(e) and 5(f)]. If we further increase $W_{0}$ to 20, then $W$ is close to an odd function of $x$. What's more, the soliton is stable and asymmetric [see Figs. $5(\mathrm{~b})$ and $5(\mathrm{c})$ ], which indicates that we can get stable soliton with near $\mathcal{P} \mathcal{T}$-symmetric Scarf-II potential by increasing the value of $W_{0}$.

In particular, for some small values of $W_{0}$, the soliton in cubic GLE is usually stable with $\alpha_{2} \leq 0$ and $\beta_{2} \geq 0$, beyond which the soliton immediately becomes extremely unstable [8]. So we investigate the relationship between $P$ and $\alpha_{2}$ with the fourth order magnetic interactions $\sigma_{2}$ [see Fig. 6]. When $\alpha_{2}>0$ and $\sigma_{2}=0, P$ of the nonlinear mode changes suddenly near $t=0$. If we fix $\sigma_{2}=0.03$, the curve about $P$ and $\alpha_{2}$ of nonlinear mode will become smooth. Thus, power of the nonlinear mode can be transformed by changing $\sigma_{2}$.

3.2 Nonlinear modes with the near $\mathcal{P} \mathcal{T}$-symmetric $\delta$-signum potential

Next, we introduce the near $\mathcal{P} \mathcal{T}$-symmetric $\delta$-signum potential

$$
\begin{aligned}
& V(x)=2 V_{0} \delta(x), \\
& W(x)=W_{0} \operatorname{sgn}(x) e^{-V_{0}|x|}-W_{1} \delta(x),
\end{aligned}
$$

to carry on our analysis, which plays a significant role in such fields as quantum physics, optics, and Bose-Einstein condensates [43,44]. 

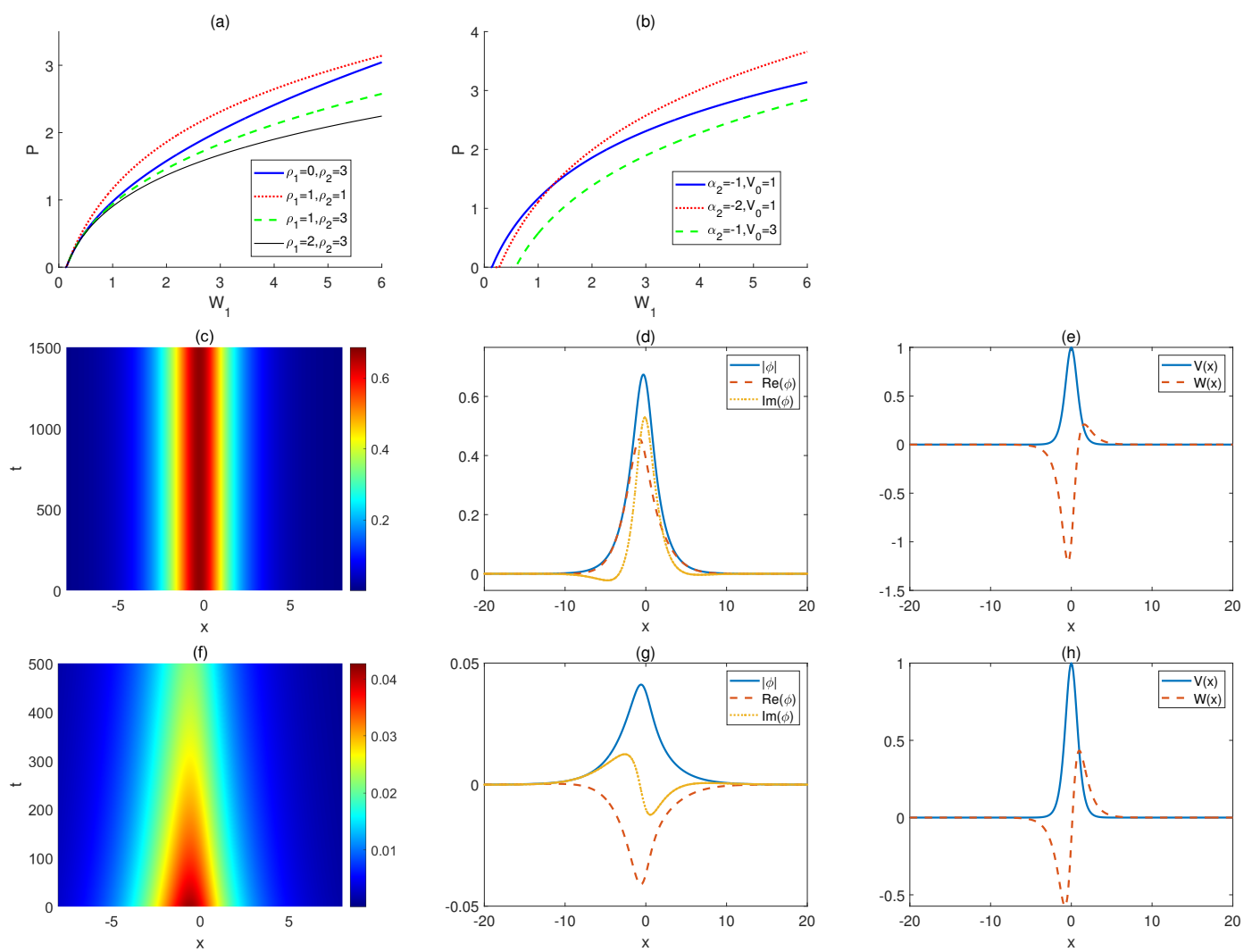

Figs. 4 Parameters are chosen as: $\alpha_{1}=1, \beta_{1}=1, \beta_{2}=1, \sigma_{1}=0.05, \sigma_{2}=0, W_{0}=1$, with(a) $\alpha_{2}=-1, V_{0}=1$, with(b) $\rho_{1}=1, \rho_{2}=1, \operatorname{with}(\mathrm{c})(\mathrm{d})(\mathrm{e}) \alpha_{2}=-1, \rho_{1}=1, \rho_{2}=1, V_{0}=1, W_{1}=1, \operatorname{with}(\mathrm{f})(\mathrm{g})(\mathrm{h}) \alpha_{2}=-1, \rho_{1}=1$, $\rho_{2}=1, V_{0}=1, W_{1}=0.14$.

The limit of the following Gaussian function can be used to express the $\delta$ function

$$
\begin{aligned}
& \delta(x)=\lim _{a \rightarrow 0^{+}} g(x ; a), \\
& g(x ; a)=\frac{\exp \left(-x^{2} / a^{2}\right)}{a \sqrt{\pi}},
\end{aligned}
$$

thus we can use the Gaussian function $g(x ; a)$ with very small parameter $a$ to approximate the $\delta$ function [43]. In order to facilitate calculations, without loss of generality, we choose $a=0.01$ in this paper.

As shown in the Figs. $7(\mathrm{a})$ and $7(\mathrm{~b})$, the horizontal coordinates is $W_{1} \in(0,6) . W_{1}$ is directly proportional to $P$. $\beta_{2}, \rho_{1}, \rho_{2}$ are inversely proportional to $P$. And when $W_{1}$ is sufficiently small, the peakon solution is becoming unstable. Results of these numerical simulation are given in Figs. $7(\mathrm{c})$ and $7(\mathrm{e})$.

When the horizontal coordinates is $W_{0} \in(-5,20)$, Fig. $8(\mathrm{a})$ shows the power of peakon solutions. When $W_{0}=0, P$ takes the minimum value. Considering $W_{0}$ as large as possible, the evolution of peakon is similar to the case under the near $\mathcal{P} \mathcal{T}$-symmetric Scarf-II potential and it experiences more than 5 periods within $1450 \leq t \leq 1500$ [see Fig. 8(b)].

Based on the above results, we consider the effect of $\beta_{2}$ and $\rho_{2}$. It is easy to show that $\beta_{2}$ and $\rho_{2}$ are inversely proportional to $P$. The two stable peakon solutions are illustrated in Figs. 9(c) 

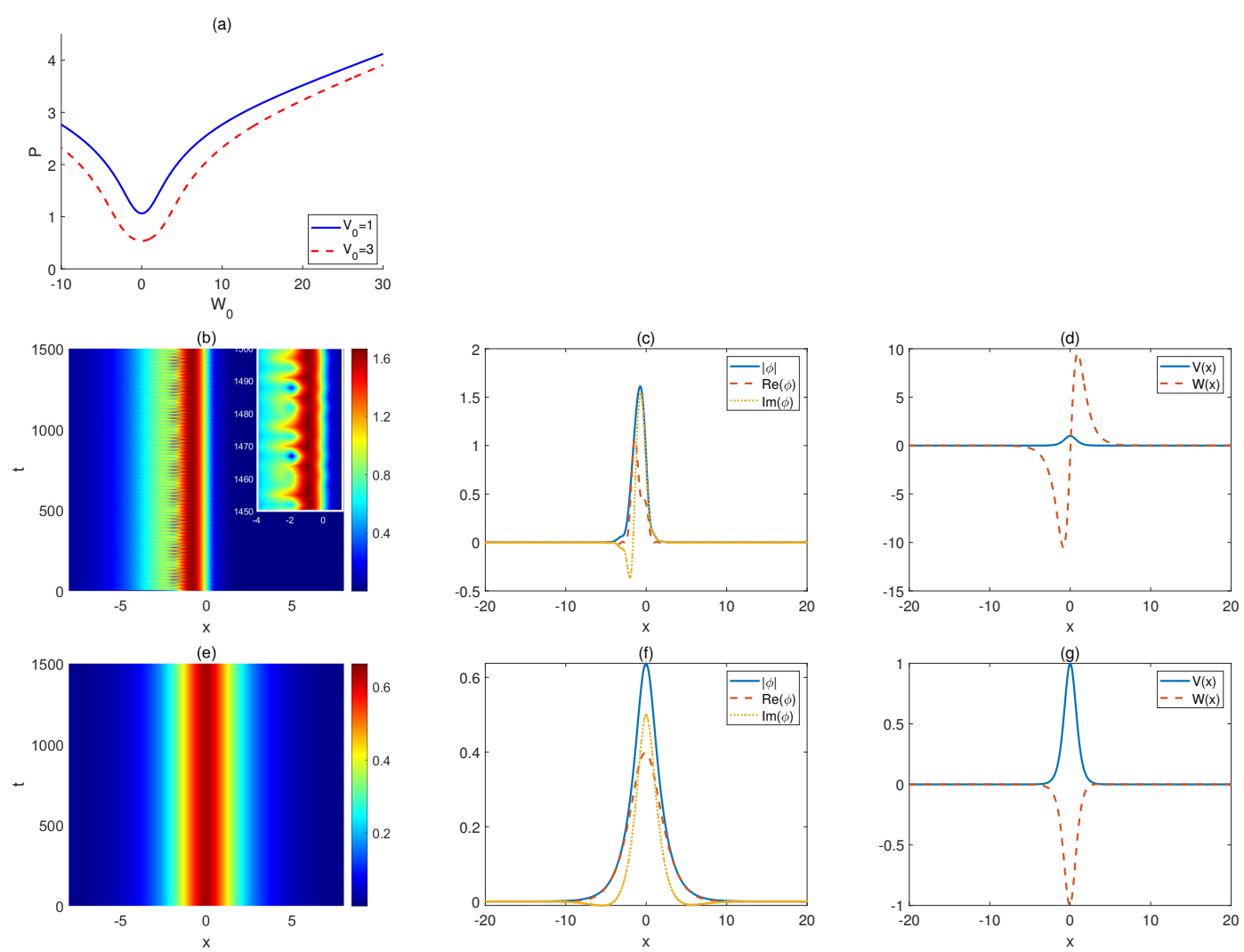

Figs. 5 Parameters are chosen as: Parameters are chosen as: $\alpha_{1}=1, \alpha_{2}=-1, \beta_{1}=1, \beta_{2}=1, \rho_{1}=1, \rho_{2}=1$, $\sigma_{1}=0.05, \sigma_{2}=0, W_{1}=1, \operatorname{with}(\mathrm{b})(\mathrm{c})(\mathrm{d}) V_{0}=1, W_{0}=20, \operatorname{with}(\mathrm{e})(\mathrm{f})(\mathrm{g}) V_{0}=1, W_{0}=0.1$.

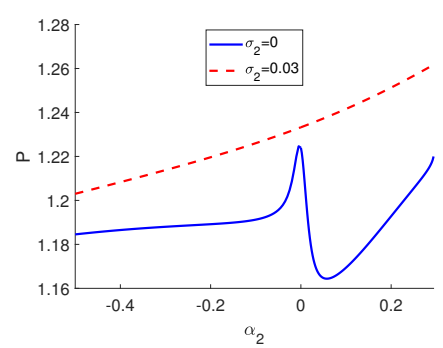

Figs. 6 Parameters are chosen as: $\alpha_{1}=1, \beta_{1}=1, \beta_{2}=1, \rho_{1}=1, \rho_{2}=1, \sigma_{1}=0.05, V_{0}=1, W_{0}=1, W_{1}=1$.

and $9(\mathrm{e})$. It can be seen that $\beta_{2}$ are related to the morphology of peakons by changing the width of nonlinear mode.

3.3 Generalized model and excitations of solitons

In this section we consider excitations of above-mentioned solitons in Eq. (15) via adiabatical change of system parameters. We restrict our interests in the following nonlinear modes with the 

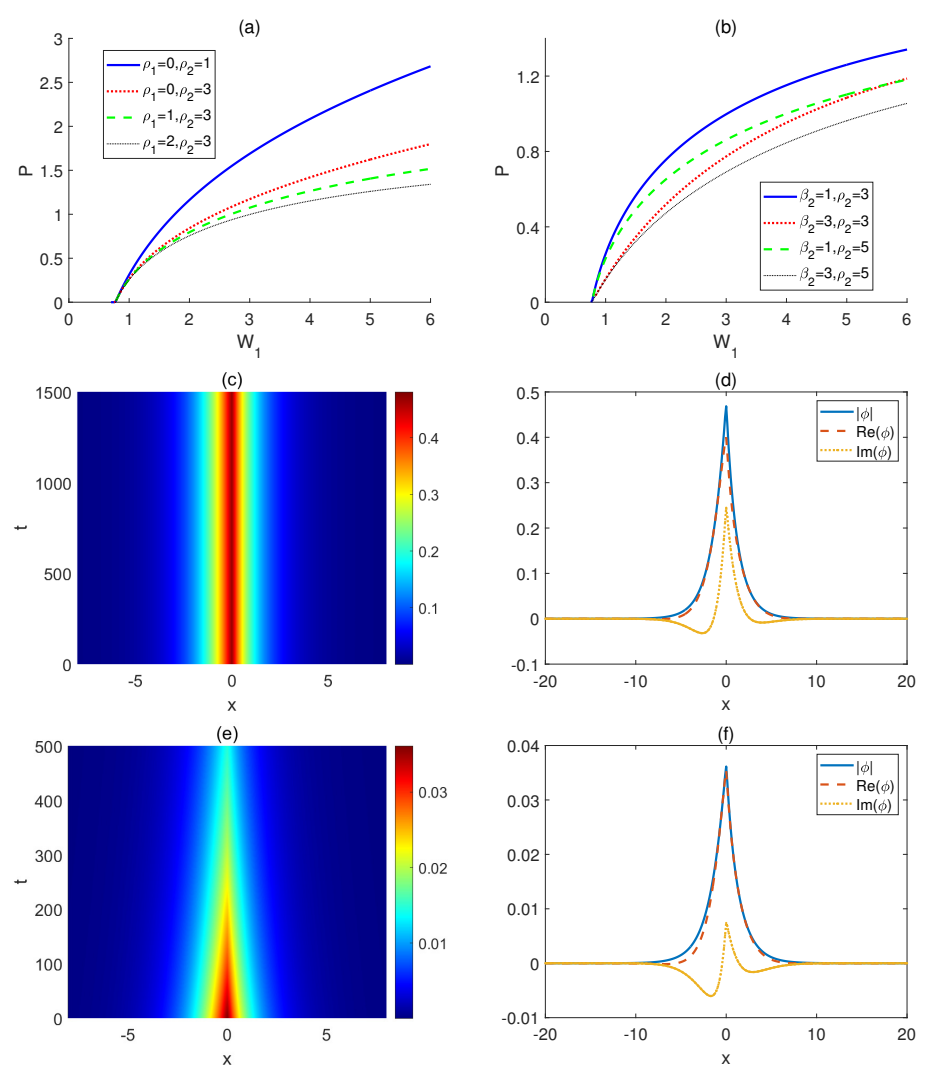

Figs. 7 Parameters are chosen as: $\alpha_{1}=1, \alpha_{2}=-1, \beta_{1}=1, \sigma_{1}=\sigma_{2}=0, V_{0}=1, W_{0}=1$, with (a) $\beta_{2}=1$, with (b) $\rho_{1}=2$, with(c)(d) $\beta_{2}=1, \rho_{1}=0, \rho_{2}=1, W_{1}=1$, with(e)(f) $\beta_{2}=1, \rho_{1}=0, \rho_{2}=1, W_{1}=0.77$.
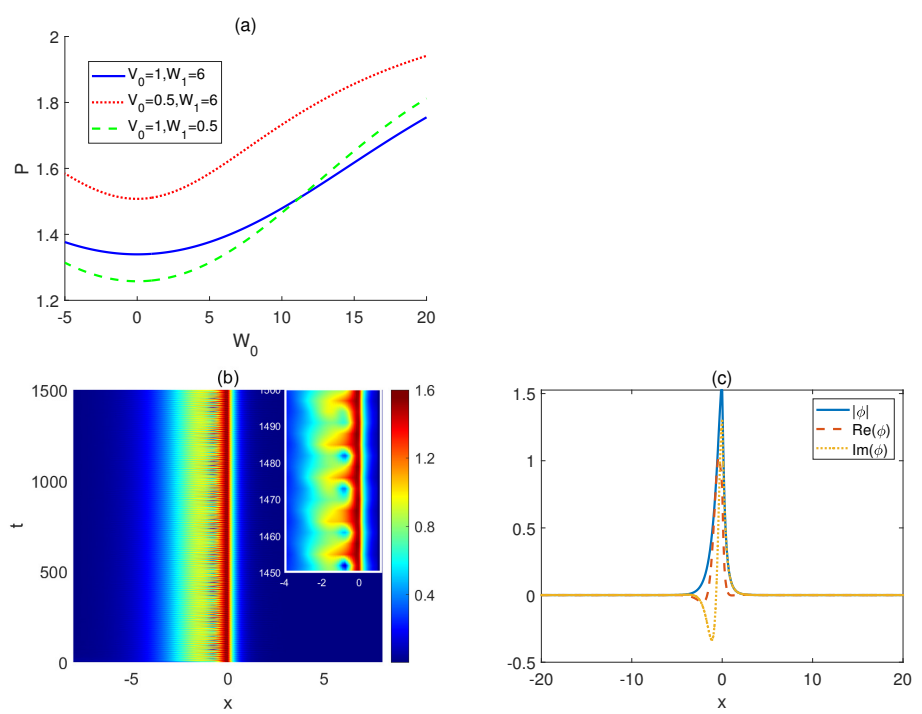

Figs. 8 Parameters are chosen as: $\alpha_{1}=1, \alpha_{2}=-1, \beta_{1}=1, \beta_{2}=1, \rho_{1}=2, \rho_{2}=3, \sigma_{1}=\sigma_{2}=0$, with(b)(c) $V_{0}=1, W_{0}=20, W_{1}=6$. 

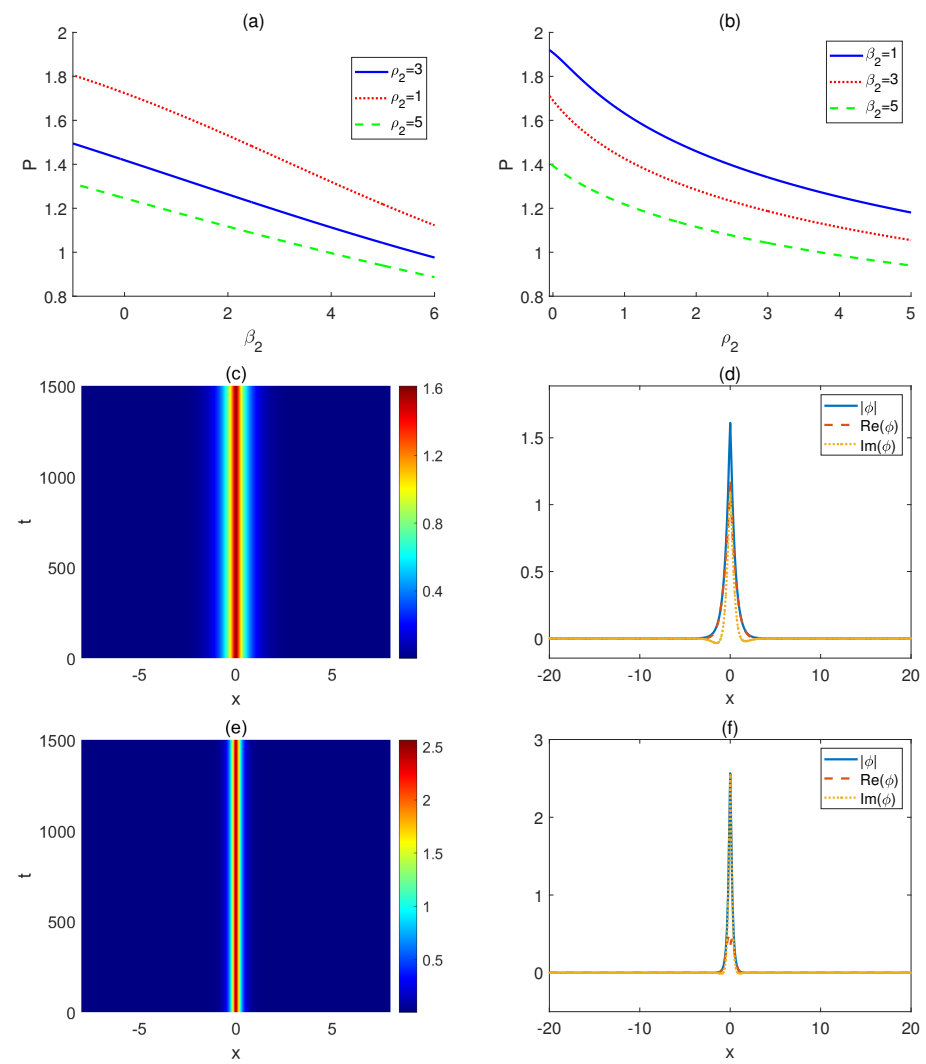

Figs. 9 Parameters are chosen as: $\alpha_{1}=1, \alpha_{2}=-1, \beta_{1}=1, \rho_{1}=2, \sigma_{1}=\sigma_{2}=0, V_{0}=1, W_{0}=1, W_{1}=6$, with(a) $\rho_{2}=3$, with(b) $\beta_{2}=1$, with(c)(d) $\beta_{2}=-1, \rho_{2}=3$, with(e)(f) $\beta_{2}=1, \rho_{2}=0$.

near $\mathcal{P} \mathcal{T}$-symmetric potentials (18) (19) and complex coefficients of Eq

$$
\begin{aligned}
& i u_{t}+\left[\alpha_{1}+i \alpha_{2}(t)\right] u_{x x}+\left[\beta_{1}+i \beta_{2}\right]|u|^{2} u+[V(x)+i W(x, t)] u \\
& +\left[\sigma_{1}+i \sigma_{2}\right] u_{x x x x}+\left[\rho_{1}+i \rho_{2}\right] u|u|^{4}=0 .
\end{aligned}
$$

In order to modulate the system parameters smoothly, we consider the following "switch-on" function:

$$
\epsilon(t)=\left\{\begin{array}{lr}
\epsilon_{1}, & t=0 \\
\frac{1}{2}\left(\epsilon_{2}-\epsilon_{1}\right)\left[1+\sin \left(\frac{\pi t}{500}-\frac{\pi}{2}\right)\right]+\epsilon_{1}, & 0<t<500 \\
\epsilon_{2}, & 500 \leq t \leq 1500
\end{array}\right.
$$

where $\epsilon_{1,2}$ respectively represent the real initial-state and final-state parameters and generate $\alpha_{2}(t)$, $W_{0}(t), W_{1}(t)$ by $\epsilon(t)$. Under the synchronous modulation of system parameters, the excitation stage and the propagation stage can be described by Eq. (21), Eq. (22). During the excitation stage $(0<t<500)$, system parameters change slowly from $\epsilon_{1}$ to $\epsilon_{2}$, and the initial state corresponding to $\epsilon_{1}$ will be adiabatically driven to the new state corresponding to $\epsilon_{2}$; during the propagation stage $(500 \leq t \leq 1500)$, system parameters are maintained at $\epsilon_{2}$, and the excited nonlinear mode will propagate in the final system. It should be noted that some system parameters can be constants [e.g., $W_{0}(x, t)=\epsilon(t)=$ const.], if we set the "switch-on" function $\epsilon(t)$ for $\epsilon_{1}=\epsilon_{2}$ [45]. 

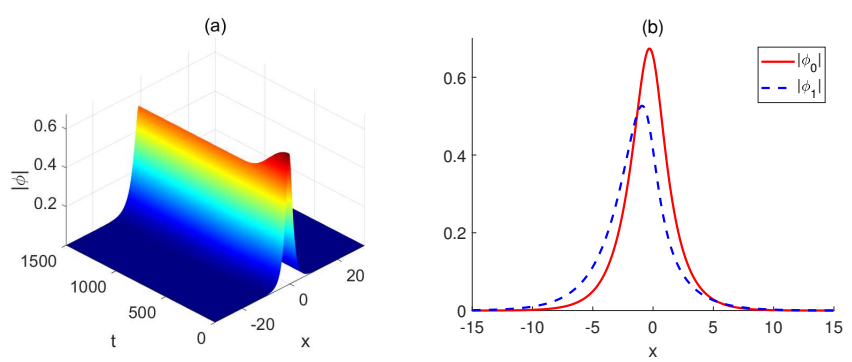

Figs. 10 Parameters are chosen as: $\alpha_{1}=1, \alpha_{2}=-1, \beta_{1}=1, \beta_{2}=1, \rho_{1}=1, \rho_{2}=1, \sigma_{1}=0.05, \sigma_{2}=0, V_{0}=1$, $W_{01}=1, W_{02}=2, W_{11}=1, W_{12}=0.14$.
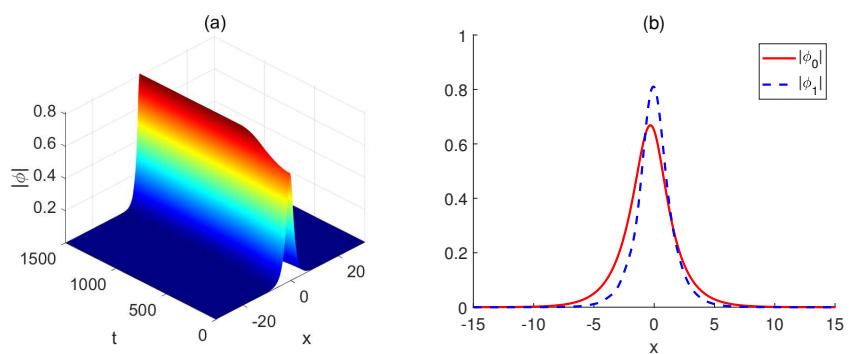

Figs. 11 Parameters are chosen as: $\alpha_{1}=1, \beta_{1}=1, \beta_{2}=1, \rho_{1}=1, \rho_{2}=1, \sigma_{1}=0.05, \sigma_{2}=0.03, V_{0}=1$, $W_{0}=1, W_{1}=1, \alpha_{21}=-1, \alpha_{22}=0$.
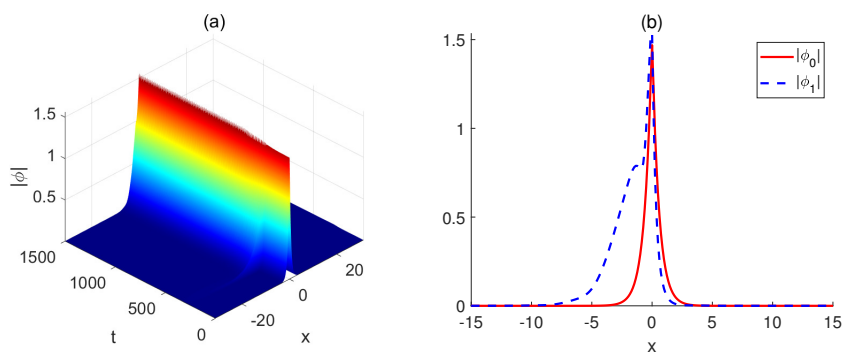

Figs. 12 Parameters are chosen as: $\alpha_{1}=1, \alpha_{2}=-1, \beta_{1}=1, \beta_{2}=1, \rho_{1}=2, \rho_{2}=3, \sigma_{1}=\sigma_{2}=0, V_{0}=1$, $W_{1}=6, W_{01}=1, W_{02}=20$

We first execute a two-parameters excitation of the soliton with the near $\mathcal{P} \mathcal{T}$-symmetric ScarfII potential. Figs. 10 shows that the the excitation of the nonlinear mode is stable with a lower amplitude with the change of $W_{0}$ and $W_{1}$, due to both the final state and initial state are stable.

Because the solutions in Fig. 6 can not be gotten directly when $\alpha_{2} \geq 0$, we consider the case in this section. As can be seen, Figs. 11 shows a stable soliton solution.

Finally, we consider the near $\mathcal{P} \mathcal{T}$-symmetric $\delta$-signum potential. We execute a single-parameter excitation of the peakon controlled by Eq. (21) via the initial condition determined. In Figs. 12, it can be seen that if $W_{01}=1$ and $W_{11}=20$, then the morphology of stable mode will change.

\section{Conclusions}

In conclusion, we study the dynamic behavior and stability of nonlinear modes in the fourth order generalized GLE with near $\mathcal{P} \mathcal{T}$-symmetric potentials. 
Firstly, we get the bilinear form of Eq. (2) by Hirota method. When the coefficients are constant and variable, analytical solutions and images of solitary wave solutions are obtained respectively. In constant coefficients, the velocity and direction of solitonic propagation phase will be changed if we choose different values of $\eta_{1}$ [see Figs. 1]. In various coefficients, the intensity of soliton is related to $\sigma_{2}(t)$ and the soliton solution is periodic when the variable coefficient $\eta_{1}(t)$ as sine function [see Figs. 2].

Secondly, we separately study the model of Eq. (15) with two novel categories of near $\mathcal{P} \mathcal{T}$ symmetric Scarf-II and $\delta$-signum potentials, and get several stable soliton and peakon solutions. Besides, the relationships between system coefficients and the power of nonlinear modes are investigated. Roughly speaking, $W_{1}$ and $P$ are positively correlated while $\rho_{1}, \rho_{2}$ and $P$ are negatively correlated [see Figs. 4(a) and 7(a)]. When $W_{0}=0$, the power of nonlinear modes takes the minimum value [see Figs. 5(a) and 8(a)]. In particular, with the near $\mathcal{P} \mathcal{T}$-symmetric Scarf-II potential, if the initial value of $W_{0}=20 W_{1}, W$ is close to an odd function of $x$ and the amplitude of nonlinear mode is periodically oscillating [see Figs. $5(\mathrm{~b})$ and $5(\mathrm{~d})$ ]. Let $W_{0}=0.1$, then $W$ approximates to an even function of $x$ and the soliton is stable and symmetric approximately [see Figs. $5(\mathrm{e})$ and $5(\mathrm{~g})$ ]. With the near $\mathcal{P} \mathcal{T}$-symmetric $\delta$-signum potential, $\beta_{2}$ is related to the morphology of peakons by changing the width of nonlinear mode and it is inversely proportional to the power of the peakon [see Figs. 9].

Finally, we analyse the excitations of nonlinear modes and get some stable cases that have not been acquired in second part. It can be seen that the morphology of stable mode is changed when we execute a single-parameter excitation of the peakon [see Figs. 11 and 12].

Acknowledgements We express our sincere thanks to all the members of our discussion group for their valuable comments.

\section{Conflict of interest}

The authors declare that they have no conflict of interest.

\section{Funding}

The authors declare that no funds, grants, or other support were received during the preparation of this manuscript.

\section{Data availability statement}

The authors declare that the manuscript has no associated data.

\section{References}

1. Ablowitz M. J., Segur H.: Solitons and the Inverse Scattering Transform. SIAM, Philadelphia (1981)

2. Ablowitz M. J., Clarkson P. A.: Solitons, Nonlinear Evolution Equations and Inverse Scattering. Cambridge university press, Cambridge (1991)

3. Musslimani Z., Makris K. G., El-Ganainy R., Christodoulides D. N.: Optical solitons in $\mathcal{P} \mathcal{T}$ periodic potentials. Phys. Rev. Lett. 100, 030402 (2008)

4. Konotop V. V., Yang J., Zezyulin D. A.: Nonlinear waves in $\mathcal{P} \mathcal{T}$-symmetric systems. Rev. Mod. Phys. 88, $035002(2016)$

5. Nozaki K., Bekki N.: Pattern Selection and Spatiotemporal Transition to Chaos in the Ginzburg-Landau Equation. Phys. Rev. Lett. 12, 2154 (1983) 
6. Haken H.: Generalized Ginzburg-Landau equations for phase transitionlike phenomena in lasers, nonlinear optics, hydrodynamics and chemical reactions. Z. Phys. B 21, 105 (1975)

7. Aranson I. S., Kramer L.: The world of the complex Ginzburg-Landau equation. Rev. Mod. Phys. 74, 99-143 (2002)

8. Chen Y., Yan Z., Liu W.: Impact of near- $\mathcal{P} \mathcal{T}$ symmetry on exciting solitons and interactions based on a complex Ginzburg-Landau model. Opt. Express, 2625 (2018)

9. Ipsen M., Kramer L., Sørensen P. G.: Amplitude equations for description of chemical reaction-diffusion systems. Phys. Rep. 337, 193-235 (2000)

10. Hecke M. van: Coherent and incoherent structures in systems described by the 1D CGLE: experiments and identification. Phys. D 174, 134-151 (2003)

11. Akhmediev N., Ankiewicz A., Soto-Crespo J.: Multisoliton solutions of the complex Ginzburg-Landau equation. Phys. Rev. Lett. 79, 4047 (1997)

12. Akhmediev N., Soto-Crespo J. M.: Exploding solitons and Shil'nikov's theorem. Phys. Lett. A 317, 287-292 (2003)

13. Skarka V., Aleksic N., Leblond H., Malomed B., Mihalache D.: Varieties of stable vortical solitons in GinzburgLandau media with radially inhomogeneous losses. Phys. Rev. Lett. 105, 213901 (2010)

14. He Y., Mihalache D.: Lattice solitons in optical media described by the complex Ginzburg-Landau model with $\mathcal{P} \mathcal{T}$-symmetric periodic potentials. Phys. Rev. A 87, 013812 (2013)

15. Soto-Crespo J. M., Akhmediev N., Chiang K. S.: Simultaneous existence of a multiplicity of stable and unstable solitons in dissipative systems. Phys. Lett. A 291, 115-123 (2001)

16. Zhang H. Q., Tian B., Meng X. H., Lü X., Liu W. J.: Conservation laws, soliton solutions and modulational instability for the higher-order dispersive nonlinear Schrödinger equation. Eur. Phys. J. B 72, 233-239 (2009)

17. Akhmediev N., Soto-Crespo J. M., Town G.: Pulsating solitons, chaotic solitons, period doubling, and pulse coexistence in mode-locked lasers: Complex Ginzburg-Landau equation approach. Phys. Rev. E 63, 056602 (2001)

18. Bender C. M., Boettcher S.: Real spectra in non-Hermitian Hamiltonians having $\mathcal{P} \mathcal{T}$ symmetry. Phys. Rev. Lett. 80, $5243(1998)$

19. Bender C. M., Brody D. C., Jones H. F.: Must a Hamiltonian be Hermitian? Am. J. Phys 71, 1095-1102 (2003)

20. Bender C, M.: Making sense of non-Hermitian Hamiltonians. Rep. Prog. Phys. 70, 947-1018 (2007)

21. Guo A., Salamo G., Duchesne D., Morandotti R., Volatier-Ravat M., Aimez V., Siviloglou G., Christodoulides

D.: Observation of $\mathcal{P} \mathcal{T}$-symmetry breaking in complex optical potentials. Phys. Rev. Lett. 103, 093902 (2009)

22. Rüter C. E., Makris K. G., El-Ganainy R., Christodoulides D. N., Segev M., Kip D.: Observation of parity-time symmetry in optics. Nat. Phys. 6, 192-195 (2010)

23. Regensburger A., Bersch C., Miri M. A., Onishchukov G., Christodoulides D. N., Peschel U.: Parity-time synthetic photonic lattices. Nature 488, 167-171 (2012)

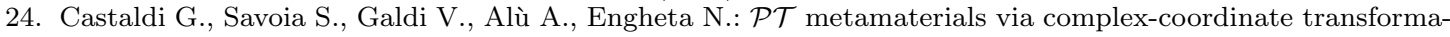
tion optics. Phys. Rev. Lett. 110, 173901 (2013)

25. Regensburger A., Miri M. A., Bersch C., Näger J., Onishchukov G., Christodoulides D. N., Peschel U.: Observation of defect states in $\mathcal{P} \mathcal{T}$-symmetric optical lattices. Phys. Rev. Lett. 110, 223902 (2013)

26. Peng B., Özdemir S. K., Lei F., Monifi F., Gianfreda M., Long G. L., Fan S., Nori F., Bender C. M., and Yang L.: Parity-time-symmetric whispering-gallery microcavities. Nat. Phys. 10, $394-398$ (2014)

27. Zyablovsky A. A., Vinogradov A. P., Pukhov A. A., Dorofeenko A. V., Lisyansky A. A.: $\mathcal{P} \mathcal{T}$-symmetry in optics. Phys. Usp. 57, 1063 (2014)

28. Chen P. Y., Jung J.: $\mathcal{P} \mathcal{T}$ Symmetry and Singularity-Enhanced Sensing Based on Photoexcited Graphene Metasurfaces. Phys. Rev. Appl 5, 064018 (2016)

29. Takata K., Notomi M.: $\mathcal{P} \mathcal{T}$-Symmetric Coupled-Resonator Waveguide Based on Buried Heterostructure Nanocavities. Phys. Rev. Appl 7, 054023 (2017)

30. Lumer Y., Plotnik Y., Rechtsman M. C., Segev M.: Nonlinearly induced $\mathcal{P} \mathcal{T}$ transition in photonic systems. Phys. Rev. Lett. 111, 263901 (2013)

31. Yan Z., Chen Y., Wen Z.: On stable solitons and interactions of the generalized Gross-Pitaevskii equation with $\mathcal{P} \mathcal{T}$-and non- $\mathcal{P} \mathcal{T}$-symmetric potentials. Chaos 26, 083109 (2016)

32. Tsoy E. N., Allayarov I. M., Abdullaev F. K.: Stable localized modes in asymmetric waveguides with gain and loss. Opt. Lett. 39, 4215-4218 (2014)

33. Konotop V. V., Zezyulin D. A.: Families of stationary modes in complex potentials. Opt. Lett. 39, 5535-5538 (2014)

34. Nixon S. D., Yang J.: Bifurcation of soliton families from linear modes in non- $\mathcal{P} \mathcal{T}$-symmetric complex potentials. Stud. Appl. Math. 136, 459-483 (2016)

35. Kominis Y.: Soliton dynamics in symmetric and non-symmetric complex potentials. Opt. Commun. 334, 265-272 (2015)

36. Kominis Y.: Dynamic power balance for nonlinear waves in unbalanced gain and loss landscapes. Phys. Rev. A $92,063849(2015)$

37. Yang J., Nixon S.: Stability of soliton families in nonlinear schrödinger equations with non-parity-timesymmetric complex potentials. Phys. Lett. A 380, 3803-3809 (2016) 
38. Zong F. D., Dai C. Q., Yang Q. and Zhang J. F.: Soliton solutions for variable coefficient nonlinear Schrödinger equation for optical fiber and their application. Acta Phys. Sin. 553805 (2006)

39. Liu W. J., Tian B., Jiang Y., Sun K., Wang P., Li M., Qu Q. X.: Soliton solutions and Bäcklund transformation for the complex Ginzburg-Landau equation. Applied Mathematics and Computation. 2017 4369-4376 (2011)

40. Musslimani Z., Makris K. G., El-Ganainy R., Christodoulides D. N.: Optical solitons in $\mathcal{P} \mathcal{T}$ periodic potentials. Phys. Rev. Lett. 100(3), 030402 (2008)

41. Hirota R.: The Direct Method in Soliton Theory. Springer, Berlin (1980)

42. Yang J.: Nonlinear waves in integrable and nonintegrable systems. SIAM (2010)

43. Chen Y., Yan Z., Mihalache D.: Stable flat-top solitons and peakons in the $\mathcal{P} \mathcal{T}$-symmetric $\delta$-signum potentials and nonlinear media. Chaos. 29, 083108 (2019)

44. Cartarius H., Wunner G.: Model of a $\mathcal{P} \mathcal{T}$-symmetric Bose-Einstein condensate in a $\delta$-function double-well potential. Phys. Rev. A 86, 013612 (2012)

45. Shen Y. J., Wen Z. C., Yan Z. Y., Hang C.: Effect of symmetry on nonlinear waves for three-wave interaction models in the quadratic nonlinear media. Chaos 28, 043104 (2018) 